\title{
LA AUTOCONSTRUCCIÓN EN UN DISTRITO DE LA PROVINCIA DE HUANCAYO, JUNÍN, PERÚ
}

\section{Self-construction in a district of the province of Huancayo, Junin, Peru}

\author{
iD Mayda Alvina Nieva Villegas ${ }^{1}$ (iD) Ketty Marilú Moscoso Paucarchuco ${ }^{2}$ \\ (iD) Stiven Roland Osorio Capcha ${ }^{3}$ (iD Manuel Michael Beraún Espíritu ${ }^{3}$ \\ 'Universidad Nacional Autónoma de Tayacaja Daniel Hernández Morillo, Perú. \\ 2Universidad Nacional Autónoma de Huanta, Perú. \\ 3Universidad Continental, Perú. \\ Correspondencia: \\ MSc. Mayda Alvina Nieva Villegas \\ maydaalvina@unat.edu.pe
}

\section{RESUMEN}

La presente investigación tiene por objeto analizar el desarrollo del complejo proceso de autoconstrucción para la ocupación de las viviendas en el distrito de Chilca Huancayo, posee un enfoque cuanti-cualitativo con diseño descriptivo, transversal retrospectivo. Se seleccionó una muestra no probabilística de los propietarios de viviendas autoconstruidas de una manzana del barrio Puzo, del distrito de Chilca. La metodología de la investigación fue de alcance analítico y método de nivel empírico, se utilizó como instrumentos de recolección de datos: fichas técnicas sobre las viviendas y entrevistas semiestructuradas a los propietarios. Los resultados mostraron que la autoconstrucción es una situación real que poco a poco se ha ido incrementando en el distrito de Chilca y es necesario conocer más sobre este proceso para proponer acciones, políticas y responsabilidades. El estudio concluyó que en el aspecto técnicoconstructivo se debe realizar asesoramiento en la materia, control de calidad de materiales de construcción, y adecuada planificación y organización de la ejecución de la obra. Además, sobre el aspecto social, hay poca información que pueda contribuir al conocimiento de la población, ya que los hábitos y costumbres definen en gran medida este proceso autoconstructivo, los jefes de familia tienen experiencia rural, donde la vivienda solo es vista como una necesidad de cobijo familiar.

Palabras clave: Autoconstrucción, vivienda, cuanti-cualitativo.

\section{ABSTRACT}

The present research aims to analyze the development of the complex process of selfconstruction for the occupation of houses in the district of Chilca - Huancayo, it has a quantitative-qualitative approach with a descriptive, cross-retrospective design. A nonprobabilistic sample of self-built homeowners was selected from a block in the Puzo neighborhood, in the Chilca district. The methodology of the project was analytical in 
scope and empirical level method, it was used as data collection instruments: technical sheets on the dwellings and semi-structured interviews with the owners. The results showed that self-construction is a real situation that has gradually increased in the Chilca district and it is necessary to know more about this process to propose actions, policies and responsibilities. The study concluded that in the technical-construction aspect, advice on the matter, quality control of construction materials, and adequate planning and organization of the execution of the work should be carried out. In addition, on the social aspect, there is little information that can contribute to the knowledge of the population, since habits and customs largely define this self-constructing process, the heads of families have rural experience, where housing is only seen as a necessity of family shelter.

Key words: Self-construction, living place, quantitative-qualitative.

\section{INTRODUCCIÓN}

En América Latina actualmente se observa que se ha dejado de lado la problemática del orden urbanístico, ya que las viviendas se vienen construyendo basados en factores económicos y sociales, sin plantear una política habitacional donde se busque dar soluciones al déficit de viviendas y fallas físicas de estas ya construidas (Mattone, 2007). Asimismo, es importante indicar que el problema habitacional es muy variante (Candón-Mena y Domínguez-Jaime, 2020), en cada país, región o ciudad, donde las condiciones de vida son muy diversas ya que esto depende de cada individuo, edad, género y también de las clases sociales donde se desenvuelven.

Al final de la primera década del siglo XX, los cambios que se vivieron en la sociedad mundial, como el crecimiento económico, la ampliación de mercados de trabajo y los nuevos mercados de consumo emergentes. Al mismo tiempo lleva a la reflexión sobre el actual cuadro urbano y habitacional, que traen cambios y también de los modelos del pasado. La economía de mercado, la industrialización, la urbanización, el crecimiento poblacional, la desigualdad social, la segregación urbana, la calidad y modalidad de acceso a la vivienda dependen de estos factores.

La autoconstrucción es la forma de edificación en este caso de una vivienda realizada mediante el trabajo directo de los propietarios o usuarios, generalmente se cuenta con el apoyo de mano de obra no calificada de familiares, vecinos - amigos, aplicando un nivel básico en la ejecución de la obra, se caracteriza por existir falla estructural, carencia de documentos, bajos a medios ingresos económicos, crecimiento familiar.

Para Pino y Ojeda (2013), la autoconstrucción tiene el único objetivo de ubicar las zonas de apropiación de terrenos, como segmento de las edificaciones informales, que se encuentra en un crecimiento acelerado de 65\% (GuillénEspallargas, 2020). Resulta importante generar programas de autoconstrucción de aplicación concertada y asistida (Romero Navarrete et al., 2005 y Álvarez y Proaño, 2010).

En Perú, existen investigaciones sobre ¿Cómo se está construyendo en Lima? ya que las consecuencias de un terremoto afectan a la sociedad y su economía conllevando a una enorme inversión en la reconstrucción de la zona afectada (García-Hernández y GinésDe la Nuez, 2020). Las diferentes ciudades del Perú crecen sin planificación como es el caso de Lima Metropolitana que viene desarrollándose sin proyección controlada incluyendo la construcción de viviendas; por ello es fundamental fortalecer el asesoramiento técnico que deben tener los usuarios de la autoconstrucción (Magliano y Perissinotti, 2020). Otro resultado en Ate, determina que $71 \%$ de las viviendas cuentan con diseños espontáneos, $57 \%$ de los propietarios consideran que sus viviendas resistirán ante una actividad sísmica y $86 \%$ construyó sin dirección técnica de un profesional (Campodonico Alcantara, 2017). 
En el plano urbano, se estructura un nuevo padrón de configuración espacial, la dispersión urbana con integración de las áreas metropolitanas (Balthazar, 2012) con referencia a la vivienda, la informalidad y la precariedad, permanecen como opción para las familias de baja renta. En las últimas décadas, la magnitud del crecimiento poblacional y la dispersión territorial, forzó al trabajador de baja renta a vivir en regiones cada vez más distantes del área central. Tal hecho, es producto de un conjunto de factores como la descentralización industrial, aceleración del proceso de urbanización, consolidación del sistema de transporte y mayor movilidad de la población. De acuerdo a Erazo Espinosa (2013), "Ios nuevos padrones corresponden a cambios que vienen a quedarse, la ciudad tradicional, de tejido continuo con límites razonables definidos, ya no es regla".

Además de los factores mencionados, la práctica de la construcción de la propia vivienda, también contribuyó para la ocupación desordenada del territorio. Las principales consecuencias de esta dispersión fueron la constitución de las regiones metropolitanas, la verticalización y la sobrepoblación de áreas ya urbanizadas, la elevación de los precios de los inmuebles urbanos y la ocupación de áreas periféricas aisladas. Como identificó Linares (2013) hace tres décadas, las ciudades continúan creciendo con base en la concentración de la renta y la segregación espacial entre ricos y pobres, con diferente distribución de recursos Municipales en sus regiones socialmente segregadas.

El exilio en la periferia, la falta de alternativas en el mercado inmobiliario formal y la carencia de recursos llevaron a muchas familias a resolver el problema de vivienda con sus manos. Sobre este tema -la autoconstrucción- desde la perspectiva de la arquitectura fue desarrollada en la presente investigación. La arquitectura porque se le considera arte, ciencia y técnica a la vez de diseñar espacios arquitectónicos y planificar el crecimiento de la ciudad de los diversos lugares y entornos en los que se desarrolla la vida material, sensitiva y espiritual en todas sus manifestaciones, individuales y colectivas. Cuya función es planificar y ordenar la ciudad tratando de impedir el desorden urbano o llamado autoconstrucción de viviendas, bajo el sistema de relación entre espacio, función y sociedad, que ocupa un lugar principal en la arquitectura. Sepuedeindicarquelas viviendasautoconstruidas han venido aumentando en las zonas urbanas como muestra el inventario habitacional (CalvoVargas, 2015 y Balthazar, 2012). En este contexto, surge el problema ¿Cómo fue el proceso de autoconstrucción de las viviendas en el distrito de Chilca - Huancayo?, determinando como objetivo principal, analizar el desarrollo del complejo proceso de autoconstrucción para la ocupación de las viviendas en el distrito.

\section{MATERIALES Y MÉTODOS}

El tipo de investigación de la presente es observacional, según el número de variables, es descriptivo. El método y diseño, es no experimental, con un alcance descriptivo analítico y método de nivel empírico. La muestra fue constituida por las viviendas autoconstruidas en una manzana del Barrio Puzo, distrito de Chilca. Los cuáles fueron realizados mediante el tipo de muestreo no probabilístico. Se utilizó como instrumentos de recolección de datos: fichas técnicas sobre las viviendas (ficha de evaluación rápida de daños de la vivienda de CONRED) y entrevistas semiestructuradas a los propietarios, por medio del cual se recopiló los datos y características cuantitativas de la unidad de análisis establecido y también se utilizó la entrevista semiestructurada, con una guía de entrevista validada por juicio de expertos para realizar el análisis FODA de la auto construcción a través de las palabras de los entrevistados.

Para el procesamiento de datos cuantitativos, se utilizó la tabla de frecuencia e histogramas estadísticos de los datos obtenidos a través de las fichas de observación contabilizados en porcentajes. Para los datos cualitativos, se utilizó el programa Atlas Ti versión 7.5, identificando y presentando las categorías analíticas apriorísticas de la autoconstrucción. Se determinó las características de las viviendas autoconstruidas y sus habitantes, en cuatro dimensiones: Falla 
estructural, crecimiento económico, bajos a medios ingresos económicos y crecimiento familiar.

Se realizó el análisis FODA, determinando las siguientes interrogantes en el análisis interno ¿Cuáles son los aspectos positivos de Ud., o su vivienda sobre el proceso de construcción? ¿Cuáles son los aspectos negativos de Ud., o su vivienda sobre el proceso de construcción?, del análisis externo ¿Cuáles son los aspectos positivos que ofrecen otras entidades (públicas o privadas) sobre el proceso de construcción de su vivienda? ¿Cuáles son los aspectos negativos que poseen otras entidades (públicas o privadas) sobre el proceso de construcción de su vivienda? El uso adecuado de la metodología permite exponer los procedimientos utilizados y trabajados para la recopilación de información que contribuyó a llegar a conclusiones acordes y valederas para la investigación.

\section{RESULTADOS}

La información recabada para el presente estudio se ordenó con la finalidad de responder a las características sociales y demográficas de los participantes.

\section{Tabla 1}

Perfil sociodemográfico de los entrevistados Rango de edades de los entrevistados

\begin{tabular}{ccc}
\hline Edad & $\mathrm{N}$ & $\%$ \\
\hline 41 & 1 & 20,0 \\
48 & 1 & 20,0 \\
56 & 1 & 20,0 \\
58 & 1 & 20,0 \\
63 & 1 & 20,0 \\
Total & 5 & 100,0 \\
\hline
\end{tabular}

Nota. Se determina el rango de edades de los propietarios de la muestra de investigación.

Observando las características de los 5 participantes, por medio de la ficha técnica aplicada, fue posible identificar que la edad varía entre 41 y 63 años, con una media de 53,2 años, con respecto al sexo de los entrevistados 80\% son mujeres y $20 \%$ varones.

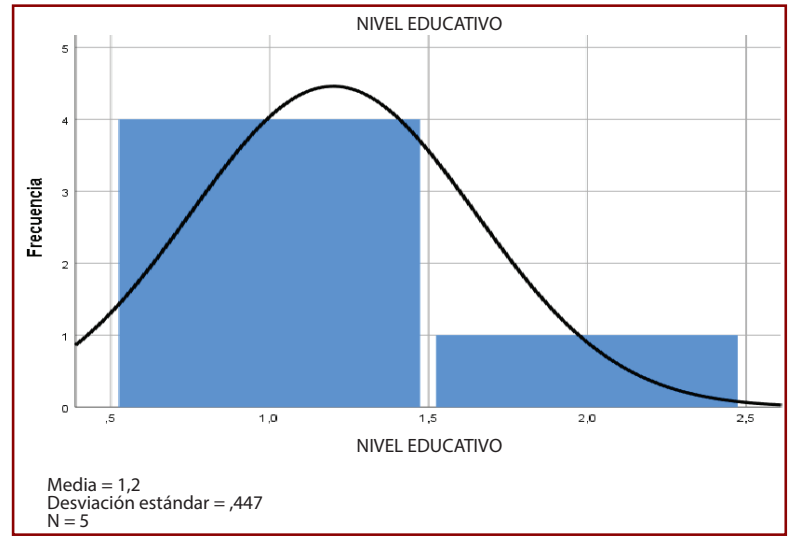

Figura 1. Perfil sociodemográfico de los entrevistados -

Nivel de escolaridad

Nota. Se determina el nivel de escolaridad de los propietarios de la muestra de investigación.

Con relación al nivel de escolaridad, 4 entrevistados (80\%) participantes tienen nivel primario completo, y solo 1 entrevistado (20\%) tienen nivel secundario completo. Todos los participantes están alejados del contexto educativo de nivel superior.

\section{Tabla 2}

Perfil sociodemográfico de los entrevistados Ocupación

\begin{tabular}{llc}
\hline \multicolumn{1}{c}{ Ocupación } & $\mathrm{N}$ & $\%$ \\
\hline Comerciante & 2 & 40,0 \\
Ama De Casa & 1 & 20,0 \\
Cosmetóloga & 1 & 20,0 \\
Obrero & 1 & 20,0 \\
Total & 5 & 100,0 \\
\hline
\end{tabular}

Nota. Se determina el nivel de ocupación de los propietarios de la muestra de investigación.

En lo que se refiere a la ocupación, 2 entrevistados (40\%) mencionan ser comerciantes, una (20\%) menciona no tener trabajo remunerado, realiza quehaceres en el dominio doméstico, una (20\%) menciona ser cosmetóloga y el 20\% restante menciona ser obrero. 
Tabla 3

\section{Nivel socioeconómico por autopercepción}

\begin{tabular}{lll}
\hline Nivel Socioeconómico & $\mathrm{N}$ & $\%$ \\
\hline Medio & 2 & 40,0 \\
Bajo & 3 & 60,0 \\
Total & 5 & 100,0 \\
\hline
\end{tabular}

Nota. Se determina el nivel de autoérsepción de los propietarios de la muestra de investigación.

En cuanto al nivel económico según autopercepción, dos entrevistados (40 \%) mencionan tener un nivel socioeconómico medio y el restante de tres entrevistados (60\%) se auto perciben con un nivel socioeconómico bajo, considerado como uno de los factores causales más importantes del autoconstrucción.

\section{Tabla 4}

\section{Perfil sociodemográfico de los entrevistados -} Crecimiento familiar

\begin{tabular}{ccc}
\hline$N^{\circ}$ de miembros de familia & N & $\%$ \\
\hline 4 & 1 & 20,0 \\
5 & 2 & 40,0 \\
7 & 1 & 20,0 \\
10 & 1 & 20,0 \\
Total & 5 & 100,0 \\
\hline
\end{tabular}

Nota. Se determina el nivel de crecimiento familiar de los propietarios de la muestra de investigación.

En cuanto, a la variable de crecimiento familiar la cantidad de miembros de familia varía entre 4 a 10 miembros por familia. Con una media de 6,2 miembros de familia, esta variable relacionada al número de habitaciones por casa, demuestra un déficit de ambientes habitables a medida que van incrementando el número de miembros de familia, según tabla a continuación:
Tabla 5

Tabla cruzada número de ambientes y número de miembros de familia

\begin{tabular}{lllllll}
\hline & \multicolumn{7}{c}{ Numero de ambientes } & & \\
& & 3 & 4 & 7 & 12 & Total \\
\hline Crecimiento familiar & 4 & 0 & 0 & 0 & 1 & 1 \\
& 5 & 1 & 1 & 0 & 0 & 2 \\
& 7 & 0 & 1 & 0 & 0 & 1 \\
Total & 10 & 0 & 0 & 1 & 0 & 1 \\
& & 1 & 2 & 1 & 1 & 5 \\
\hline
\end{tabular}

Nota. Se determina el cruce entre el número de ambientes y crecimeinto familiar.

En la siguiente tabla se observa que no hay relación directa o indirectamente proporcional entre los números de ambientes y el crecimiento familiar, de tal modo que pueden existir cuatro miembros de una familia y 12 ambientes disponibles, y 10 miembros de familia y 7 ambientes disponibles.

\section{Tabla 6}

Numero de niveles por vivienda

\begin{tabular}{lll}
\hline $\mathrm{N}^{\circ}$ De Pisos & $\mathrm{N}$ & $\%$ \\
\hline 2 & 4 & 80,0 \\
3 & 1 & 20,0 \\
Total & 5 & 100,0 \\
\hline
\end{tabular}

Nota. Se determina la cantidad de niveles por vivienda.

En lo que respecta al número de pisos por vivienda se observa que solo uno (20\%) vivienda tiene tres niveles y el restante cuatro viviendas (80\%) presentan dos niveles.

\section{Tabla 7}

\section{Tipo de uso de vivienda}

\begin{tabular}{lll}
\hline Tipo de vivendia & $\mathrm{N}$ & $\%$ \\
\hline Comercial & 1 & 20,0 \\
Multifamiliar & 1 & 20,0 \\
Unifamiliar & 3 & 60,0 \\
Total & 5 & 100,0 \\
\hline
\end{tabular}

Nota. Se determina el tipo de vivienda, entre comercial, multifamiliar y unifamiliar. 
En lo que respeta al uso de la vivienda autoconstruida, se observa en la tabla que una vivienda (20\%) es de uso comercial principalmente, mientras que una (20\%) es usada como vivienda multifamiliar para varios hermanos con sus respectivas familias y las tres viviendas restantes (60\%) manifiestan que son de uso unifamiliar, pero en todos los casos se observó que las viviendas son acondicionadas para usos comerciales: taller de soldadura, salón de belleza, tienda, etc., esto concuerda con la discusión teórica del uso capitalista del bien inmueble.

\section{Perfil de la autoconstrucción}

En esta segunda parte, se analizaron los discursos de los propietarios de las viviendas autoconstruidas en las siguientes dimensiones: antecedentes, recursos económicos, crecimiento familiar, responsabilidad municipal, daños, deseos, descontento.

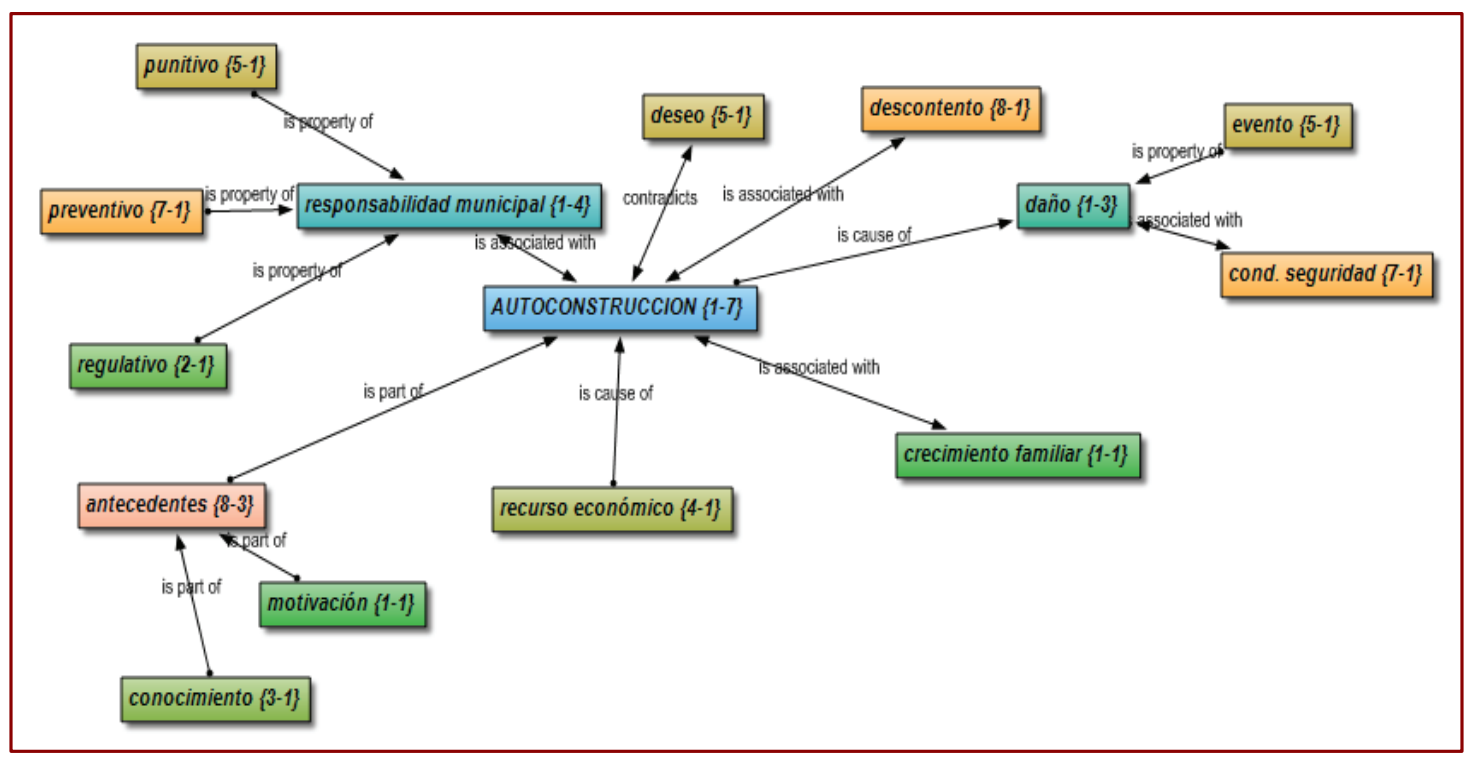

Figura 2. Perfil de la autoconstrucción.

Nota. Palabras más usadas de acuerdo al perfil de la autoconstrución.

Por lo general las viviendas autoconstruidas fueron edificadas hace más de 20 años, por iniciativa de los propietarios, por la necesidad de vivir, orientadas únicamente por los albañiles de entonces. Desconociendo los trámites administrativos ante la municipalidad de su jurisdicción.

"...Esta vivienda lo construí hace más o menos 25 años. Me animé a construir para poder vivir."

"... yo hice a mi criterio aquí quiero mi sala, mi cocina, mi tienda y cuartos para alquilarme, el albañil me dio el alcance de los materiales" "...por la necesidad de vivir, porque no tenía cuartos, vivía en un cuarto alquilado, no tenía donde vivir, a veces la dueña se cansaba me decía, hay ya estas ensuciando, por eso como sea me compré mi terrenito y como sea me construí mi casita" 


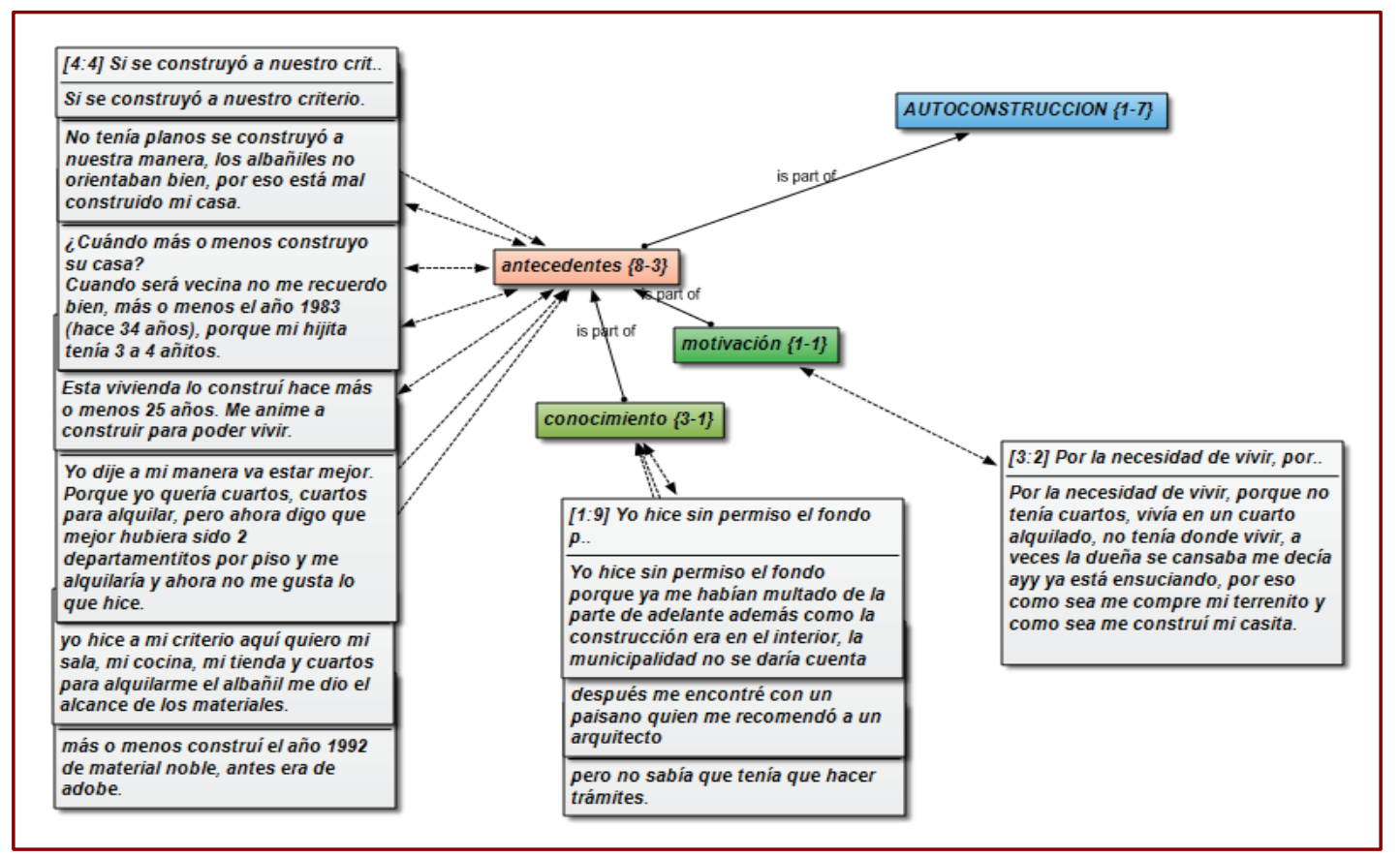

Figura 3. Antecedentes de la autoconstrucción

Nota. Frases más usadas respecto a los antecedentes de la autoconstrucción.

El tema del recurso económico jugó un papel importante para edificar viviendas autoconstruidas, ya que en su mayoría de los entrevistados señalo que no contaban con el dinero para contratar un profesional, para la dirección técnica de sus viviendas. Asimismo, es importante señalar que, en esos años, los profesionales en la materia, arquitectos e ingenieros civiles eran muy pocos y los trabajos que realizaban tenía un costo mayor a la actualidad.

“...no tenía posibilidades para yo poder contratar un profesional para que me guie, porque no había plata me faltaba dinero" "...el Arq. Arias me dijo. Ahora lo vamos actualizar no más, pero si vas hacer más construcción vuelves para diseñarlo. Pero como me había cobrado bien caro, como ahora cobrarme 5,000 mil así me cobro. Por eso no fui."

La autoconstrucción también está muy asociada al crecimiento familiar porque debido a ello se vuelve en una gran necesidad de habitar, ya que venían viviendo en cuartos alquilados, sin espacio necesario para el ambiente de una sala, cocina, comedor, dormitorios. Ya que todas sus actividades tenían que realizarlo prácticamente en un solo ambiente, asimismo, se considera el uso de los servicios higiénicos compartidos con los demás inquilinos de la casa. "... urgencia de poder vivir"

La responsabilidad municipal se puede señalar que está asociada a la autoconstrucción debido que cumple roles como, el preventivo, el regulativo y el punitivo. Pero, el incumplimiento de estos o un cumplimiento inoportuno ayuda a generar viviendas autoconstruidas, debido que la municipalidad no realiza la notificación oportuna al inicio de la construcción, sino cuando ya están construidas, casi siempre sin respetar el plan de desarrollo urbano del distrito, no respetando las secciones de las calles. Por ello, se viene generando pasajes o callejones. Asimismo, cumplen un rol punitivo, pero se debería priorizar un buen trabajo preventivo y con ellos orientar a los propietarios de los pasos a seguir para obtener mejores resultados.

"... ¿y cuando empezaste a construir alguien de la 
municipalidad se acercó? No vino, hasta cuando ya estaba vaciado el primer piso"

"...sería bueno que envíen a un profesional para que nos pueda asesorar antes de construir nuestras casas"

"... vinieron cuando ya estábamos construyendo la pared del segundo piso, ahí vino a ponernos multa".

Los eventos ocurridos en el área de trabajo, comúnmente se da las inundaciones en épocas de lluvia, como inundaciones con agua de lluvia y también el retorno a las casas de las aguas de desagüe también de las consecuencias en las infraestructuras como rajaduras, fisuras en muros, techos, corrosión de la pintura por la humedad del techo, etc.

"... Por construir en partes el techo esta parchado y existe rajaduras y fisuras"

"... Hay algunas fallas con rajaduras filtración de agua se está descascarando la pintura"

El deseo de los propietarios en volver a construir sus viviendas con el asesoramiento y la participación de un profesional de la carrera, realizar un proyecto completo, contar con la licencia de construcción - modalidad obra nueva y no de regularización. Controlar el uso de buenos y adecuados materiales para la ejecución de la obra. Contar con un buen diseño tanto del exterior y el interior de sus viviendas. Buscando espacios cómodos y adecuados para el uso de los propietarios y sus familias.

"... si lo volvería hacer, aunque sacando préstamo de un banco lo que sea. A veces pienso tumbarlo..."

"... Creo que hubiera sido mejor construir con el asesoramiento de un arquitecto o un profesional"

"... yo haría tumbar esa casa y volvería hacer de nuevo"

La autoconstrucción conlleva al descontento de los propietarios con sus viviendas en cuanto a su forma y fondo, o sea su fachada y la distribución de sus ambientes. Cuando debería ser lo contrario, una vivienda que cuente con ambientes adecuados para el tipo de actividades a realizar. Donde el propietario se sienta cómodo y contento. Como comúnmente lo denominamos: como en casa. Asimismo, es importante señalar el descontento de los vecinos debido a los problemas que vienen sufriendo. : "con esta experiencia que yo tengo quizá hubiera hecho mi casa bien, aunque chiquito, de que vale que hecho algo grandazo toda una chanfainita" "... no me gusta que el desagüe no hay donde salir"

\section{DISCUSIÓN}

El factor decisivo para autoconstruir una vivienda, es: la posibilidad de inversión de acuerdo sus recursos económicos, al menor costo. Además, de una perspectiva cultural, según (Varela, 2017 y Hernández-Flores, 2017) la práctica de autoconstrucción parece ser parte de la tradición de las familias de los entrevistados, pues la mayoría manifiesta que habían construido sus propias casas en el lugar de origen, por lo que es considerado una buena alternativa para la obtención de la casa propia.

La actitud revelada por los entrevistados en la opción de autoconstrucción contrasta con lo observado por (Moya Fuentes et al., 2017), sobre el acceso a la habitación popular en la década de 1930, donde la construcción civil aun no era una tradición entre los trabajadores urbanos, este hecho, hacía que la tarea de la casa propia sea tarea de los propietarios. Durante las entrevistas se evidenció que la actividad de autoconstrucción es una actividad independiente de consejos externos.

Así, la autoconstrucción fue tomada como el modo más rápido de acceso a la vivienda por las clases sociales más populares (Ferro, 2006), donde la ideología de la casa propia fue tan ampliamente difundida que se convirtió en un pensamiento concreto. Este hecho también fue observado en las entrevistas y corroborado por las observaciones de (Jiménez, 2020). Además, de la importancia real de propiedad de un inmueble, fue posible percibir los sentimientos de satisfacción y solidaridad expresados por la mayoría de los entrevistados (Bonduki, 2009).

Al respecto de la satisfacción, hay un fundamento en el punto de vista de García-Hernández y Ginés-De la Nuez, (2020), según el cual plantea la siguiente pregunta: ¿el proceso autónomo 
de provisión habitacional tiene un importante significado para los habitantes? puede ser que sí, pero Balthazar (2012) cuestiona el motivo por el cual, la participación o control es indispensable solo por los pobres, en cuanto la clase media se aloja cada vez más en apartamentos producido por grandes empresas y parecen satisfechas con eso. La satisfacción entonces resultaría de: ¿el proceso autónomo de provisión o de conquista de la casa propia? No hay un parámetro en la presente investigación que responda a esta pregunta. Pero si puede ser considerada una variable para estudios posteriores.

La autoconstrucción, como fue observada, se desenvuelve de modo preponderante en el territorio, pues, atiende a propósitos de una minoría dominante, interesada en mantener los privilegios de localización y de infraestructura urbana. En concordancia con tal observación, Maricato (2011, p.3) afirma: "una parte de la ciudad es hecha ilegalmente, por las manos de los habitantes, ¿para qué? Para mantener el mercado como él es. Para mantener la propiedad inmobiliaria como ella es y para mantener la sociedad patrimonialista como ella es".

\section{CONCLUSIONES}

- El padrón habitacional es resultado de la articulan las relaciones de las diversas clases sociales y también del modelo de actuación del estado en el que se debe garantizar los derechos sociales, entre ellos la vivienda, se resalta la responsabilidad preventiva e informativa que deben desenvolver los municipios tanto distritales como provinciales, más que la aplicación de la función punitiva, que es multar a quien construyó sin licencias respectivas.

- Los propietarios no aprueban sus proyectos habitacionales en los órganos institucionales, y no tienen el aval para realizar la construcción. En estas condiciones las viviendas están sujetas a multas y embargos. A estos factores se suman la baja calidad de habitabilidad y la explotación económica de los habitantes de estas viviendas, por la falta de conocimiento técnico y falta de remuneración en el esfuerzo desprendido por construir su propia casa.

- El aspecto técnico-constructivo de las autoconstrucciones, se puede manifestar que no fueron realizadas con el asesoramiento de un profesional en la materia, dejando de lado el control de la buena calidad de los materiales de construcción, la adecuada planificación y organización de la ejecución de la obra.

- Sobre las consecuencias de la autoconstrucción serán divididas en el análisis FODA, porque los propietarios manifiestan que es una FORTALEZA el apoyo de los vecinos en la construcción de sus viviendas, como una forma de conservación de las costumbres; además, los propietarios manifiestan el deseo de volver a construir adecuadamente sus viviendas, siempre y cuando, tuvieran la posibilidad de contar con recursos económicos. Como OPORTUNIDADES no identificaron políticas municipales que puedan prevenir la autoconstrucción, o puedan orientar a los nuevos propietarios a construir de forma ordenada sus viviendas. Como DEBILIDAD prima el desconocimiento de las técnicas y procedimientos para la construcción de viviendas adecuadamente habilitadas para su uso, pues las actuales viviendas cuentan con diversas dificultades y daños en su estructura que imposibilitan la morada, produciendo descontento sobre sus viviendas entre los propietarios; y como AMENAZA son las políticas punitivas municipales sobre la autoconstrucción que lo único que hacen es multar a los propietarios de las viviendas autoconstruidas, pero no previenen $u$ orientan la adecuada construcción de viviendas, pues los propietarios manifiestan que si, al momento de iniciar la construcción de sus viviendas hubieran recibido el apoyo técnico de los profesionales de la municipalidad, hubieran construido una vivienda mejor y habitable.

\section{RECOMENDACIONES}

Es importante coordinar con los colegios profesionales y las municipalidades, para iniciar o plantear programas de asesoría técnica para la autoconstrucción de viviendas, como parte de un 
trabajo de proyección y extensión de servicio a la sociedad, evitando el rol punitivo, fortaleciendo las actividades preventivas y promover políticas interculturales de la práctica profesional, respetando costumbres y tradiciones comunales, en la ejecución de las obras, como el ayni.

\section{REFERENCIAS BIBLIOGRÁFICAS}

Álvarez, P., y Proaño, D. (2010). La vivienda económica : aproximación desde la arquitectura. Arquitectura y Urbanismo, 256. Disponible en: http://dspace.ucuenca.edu. ec/bitstream/123456789/2489/1/tm4295. pdf

Balthazar, R. D. S. (2012). A permanência da autoconstrução : um estudo de sua prática no Município de Vargem Grande Paulista. Disponible en: https://teses.usp.br/teses/ disponiveis/16/16137/tde-02072012132335/publico/dissertacao_renata.pdf

Bonduki, N. (2009). Do Projeto Moradia ao programa Minha Casa, Minha Vida. Teoria e Debate, 8-14. Disponible en: https:// teoriaedebate.org.br/2009/05/01/doprojeto-moradia-ao-programa-minha-casaminha-vida/

Calvo-Vargas, M.-H. (2015). PROYECTO DE VIVIENDA MULTIFAMILIAR DE UNA EMPRESA NUEVA CON ENFOQUE EN REDUCIR LA BARRERA DE INGRESO MEDIANTE LA AUTOCONSTRUCCIÓN (Vol. 2015) [Universidad Católica del Perú]. Disponible en: http://tesis.pucp.edu.pe/repositorio/ bitstream/handle/20.500.12404/6375/ C A LV O _ G R O V E R _ M ATAY O S H I _ ALFREDO_PROYECTO_MULTIFAMILIAR. pdf? sequence $=1$ \&isAllowed $=y$

Campodonico Alcantara, T. M. (2017). Evaluación de los Problemas de Ubicación y Configuración Estructural en Viviendas Autoconstruidas en la Comunidad Urbana Autogestionaria de Huaycán, Ate, Lima, 2017 [Universidad Cesar Vallejo]. In UCV. Disponible en: https://repositorio.ucv.edu. pe/ bitstream/handle/20.500.12692/11890/ C a m p o d o n i c o _ A T M. pdf? sequence $=1$ \&isAllowed $=y$

Candón-Mena, J., y Domínguez-Jaime, P. (2020). La Autoconstrucción De Viviendas En Marinaleda Desde La Perspectiva Del Gobierno De Los Bienes Comunes De Ostrom. Acme, 19(3), 684-706. Disponible en: https:// hdl.handle.net/11441/103292

Erazo Espinosa, J. (2013). INFRAESTRUCTURAS URBANAS EN AMÉRICA LATINA: GESTIÓN Y CONSTRUCCIÓN DE SERVICIOS Y OBRAS PÚBLICAS. Disponible en: https://edisciplinas. usp.br/pluginfile.php/5578303/mod_ resource/content/0/Erazo\%2C\%202013.pdf

Ferro, S. (2006). Do Projeto Moradia ao programa Minha Casa, Minha Vida. Teoria e Debate, 8-14. Disponible en: https://teoriaedebate. org.br/2009/05/01/do-projeto-moradia-aoprograma-minha-casa-minha-vida/

Bonduki, N. (2009). Nota sobre "O vício da virtude." Novos Estudos - CEBRAP, 76, 229- 234. doi: $\quad$ https://doi.org/10.1590/S010133002006000300012

García-Hernández, J. S., y Ginés-De la Nuez, C. (2020). Geografías de la desposesión en la ciudad neoliberal: Ejecuciones hipotecarias y vulnerabilidad social en santa cruz de tenerife (canarias-españa). Eure, 46(138), 215-234. doi: https://doi.org/10.4067/S025071612020000200215

Guillén-Espallargas, G. (2020). Sembrando en las lindes. Aproximación al análisis de las relaciones entre autocultivo y autoconstrucción en la Barcelona del s. XX. UPCcommons. Disponible en: https://upcommons. upc.edu/bitstream/ handle/2117/328715/

Hernández-Flores, J. Á. (2017). Reseñas y comentarios bibliográficos. Ortega, Liliana (2016), Autoconstrucción de vivienda, espacio y vidad familiar en 
la Ciudad de México. Cuadernos de Economía, 14(20),313-318. doi: https://doi. org/10.24201/edu.v32i3.1718

Jiménez, R. (2020). Análisis de vigencia del sistema constructivo LAD-MA para la autoconstrucción asistida de viviendas progresivas. Informes de La Construcción, 72(560), e367. doi: https://doi.org/10.3989/ ic. 73728

Linares, S. (2013). LAS CONSECUENCIAS DE LA SEGREGACIÓN SOCIOESPACIAL: UN ANÁLISIS EMPÍRICO SOBRE TRES CIUDADES MEDIAS BONAERENSES (OLAVARRÍA, PERGAMINO Y TANDIL). Redalyc, 14, 27. Disponible en: https://www.redalyc.org/ articulo.oa?id=369233934001

Magliano, M. J., y Perissinotti, M. V. (2020). La periferia autoconstruida: Migraciones, informalidad y segregación urbana en argentina.Eure,46(138),5-23.doi:https://doi. org/10.4067/S0250-71612020000200005

Mattone, R. (2007). Investigación y formación para la evolución de las tradiciones. Los bloques perfilados para la autoconstrucción. Apuntes: Revista de Estudios Sobre Patrimonio Cultural Journal of Cultural Heritage Studies, 20(2), 318-323. Disponible en: https:// www. mendeley.com/catalogue/07768150357d-3755-bb10-13a1d598f860/?utm_ s o u r c e $=$ d e s k t o p \& u t m _ medium=1.19.8\&utm_campaign=open_ca talog\&userDocumentld=\%7B8e141850adb6-4e79-a80c-400d16b26bfd\%7D

Moya Fuentes, M., Bia Platas, A., Carrasco Andrino, M., Jiménez Pascual, M., Ramón Martín, A., y SolerGarcía, C. (2017).3779_Memoria Red en metodologías docentes con TICS 2016/2017: implemen- tación de la plataforma virtual Google Classroom. Memorias Del Programa de Redes-I 3 CE De Calidad, Innovación e Investigación En Docencia Universitaria. Convocatoria 2016-17, 2564-2574. www. cedro.org. Disponible en: http://rua.ua.es/ dspace/handle/10045/73595
Pino, A., y Ojeda, L. (2013). Ciudad y hábitat informal: Las tomas de terreno y la autoconstrucción en las quebradas de Valparaíso. Revista INVI, 28(78), 109-142. doi: https://doi.org/10.4067/invi.v0i0.660

Romero Navarrete, L., Hernández Rodríguez, M., y Acevedo Dávila, J. (2005). Vivienda y autoconstrucción. Participación femenina en un proyecto asistido. Frontera Norte, 17(33), 107-131. Disponible en: http:// www.scielo.org.mx/scielo.php?script=sci_ arttext\&pid=S0187-73722005000100005

Varela, L. D. (2017). La cooperativa copacabana y el barrio la asunción. de la erradicación de la villa 31 a la autoconstrucción de vivienda durante la última dictadura militar argentina (1976-1983). URBANA: Revista Eletrônica Do Centro Interdisciplinar de Estudos Sobre a Cidade, 9(1), 166. doi: https://doi. org/10.20396/ urbana.v9i1.8647226 